\title{
Etude de l'absorption du technétium 99 par le riz irrigué (Oryza sativa L.)*
}

\author{
C.M. VANDECASTEELE ${ }^{\star \star}$, R. DE BECKER ${ }^{\star \star}$, TANG VAN HAI*** \\ et C. MYTTENAERE ${ }^{\star \star \star \star}$ \\ (Manuscrit reçu le 2 septembre 1982)
}

\begin{abstract}
RÉSUMÉ
L'absorption du technétium, radionucléide artificiel essentiellement issu du cycle du combustible nucléaire, a été étudiée chez le riz (Oryza sativa L.) en aquiculture et en sol irrigué par une solution contaminée.

Les cinétiques d'absorption en flux continu pour différentes concentrations en ${ }^{99} \mathrm{Tc}\left(0,017\right.$ à $17 \mu \mathrm{Ci}$. $\left.\mathrm{I}^{-1}\right)$ révèlent l'existence de deux phases : la première à dominance passive (diffusion dans les espaces libres) et la seconde, linéaire, résultant de la pénétration du technétium dans les cellules et de ses conséquences.
\end{abstract}

L'évolution de la désorption met en évidence la présence de deux compartiments dont le premier correspondrait aux "espaces libres apparents" tandis que le second, comptant plus des $95 \%$ de l'activité de la plante, représenterait la fraction absorbée. La demi-vie biologique dans ce second compartiment est telle ( $\mathbf{4 0}$ à $\mathbf{5 0}$ jours) qu'en cas de pollution accidentelle, une décontamination totale des plantes ne peut être attendue.

Comme les essais en aquiculture, les essais en sol montrent l'apparition de symptômes de toxicité pour une concentration dans la solution d'irrigation de $17 \mu \mathrm{Ci}^{-\mathrm{I}^{-}}$. Les facteurs de transfert calculés sont très élevés, de l'ordre de 1000 pour les parties aériennes végétatives. La répartition du technétium entre les différentes parties de la plante varie selon la concentration en ${ }^{99} \mathrm{Tc}$ mais plus de $90 \%$ sont localisés dans les parties aériennes; les caryopses (parties comestibles) n'en renferment que $1 \%$.

\section{ABSTRACT}

The absorption of technetium 99 (an important fission product which can be released in the environment at different steps of the nuclear fuel cycle) by rice (Oryza sativa L.) was studied in nutrient solutions and in flooded solls with contaminated water.

*Publication n 1941 du programme Biologie-Radioprotection CCE DG XII.

**Université Catholique de Louvain, Unité de physiologie végétale, Place Croix du Sud, 4 B-1348 Louvain-La-Neuve, Belgique.

**Université Catholique de Louvain, Département des sciences du sol, Place Croix du Sud, 2 B-1348 Louvain-La-Neuve, Belgique.

***Programme Biologie - Radioprotection, CCE Direction générale XII, Commission des communautés européennes, Rue de la Loi, 200 B-1049 Bruxelles, Belgique. 
The absorption kinetics established in water culture (continuous flowing system) for ${ }^{99} \mathrm{Tc}$ concentrations ranging from 0.017 to $17 \mu \mathrm{Ci}$. $\mathrm{I}^{-1}$ revealed two phases : the former corresponds to the diffusion in the apparent free spaces and the second, which is linear, represents the transfer of ${ }^{99} \mathrm{Tc}$ from the external medium into the root cells as well as its translocation to the leaves.

The study of the desorption mechanism confirmed the existence of these compartments, the second one containing more than $95 \%$ of the total activity of the plant. The biological half-life of ${ }^{99} \mathrm{Tc}$ of the second compartment is so high that decontamination of the plant may not be expected.

In soils, toxicity symptoms were observed for a ${ }^{90} \mathrm{Tc}$ water concentration of $17 \mu \mathrm{Ci} . \mathrm{I}^{-1}$. The transfer factors calculated in irrigated soils are very high $\left(>10^{3}\right.$ for the leafy shoots) and the distribution of ${ }^{99} \mathrm{Tc}$ between the different organs varies with the concentration used. More than $90 \%$ of ${ }^{99} \mathrm{Tc}$ is found in the leafy shoots meanwhile $1 \%$ of the plant total activity is only found in the caryopses.

\section{INTRODUCTION}

L'ingestion de nourriture contaminée constitue, dans le cas de radionucléides de demi-vie très longue, une voie importante du transfert vers l'homme de la radiocontamination du milieu. Toutefois, les modèles développés pour décrire le cheminement de ces éléments de la source à l'homme, sont grevés de nombreuses incertitudes, et certaines voies de transfert, en l'occurence l'irrigation par submersion, sont actuellement très mal connues.

La détermination des paramètres des différents modèles, tant à court terme qu'à long terme, nécessite des études fondamentales destinées à améliorer la compréhension des phénomènes; celles-ci exigent de longs délais et doivent être entreprises dès maintenant, même si les problèmes pratiques ne se posent pas encore.

L'accumulation par le riz de divers polluants, tant nucléaires que conventionnels, a déjà fait l'objet de nombreuses études $[2,10,12]$ qui ont démontré le rôle non négligeable joué par l'eau d'irrigation dans le transfert de ces éléments vers la plante en conditions de rizière irriguée par submersion.

Par ailleurs, plusieurs travaux menés durant ces dernières années ont mis en évidence l'importance du technétium en tant que radionucléide issu du cycle du combustible nucléaire [1,5], et essentiellement de ${ }^{99} \mathrm{Tc}$ qui, seul parmi ses différents isotopes, tous radioactifs, apparaît, de par sa très longue demi-vie $\left[2,12.10^{5}\right.$ ans $]$ et son rendement de fission élevé $[6,3 \%]$, comme un risque potentiel pour l'homme et son environnement $[20,22]$.

Les résultats acquis jusqu'à présent $[3,8,9,13]$ montrent qu'en solution aqueuse, de même qu'en sol bien aéré et en l'absence d'agents réducteurs puissants, cet élément prédomine sous une forme anionique oxydée $\left[\mathrm{TcO}_{4}^{-}\right.$: ion pertechnétate] très soluble, très mobile et hautement bioassimilable [21].

Plusieurs auteurs ont étudié sa rétention au niveau des sols et l'ont traduite par des coefficients de distribution [Kd] qui se sont révélés relativement faibles $[19,22,23]$ exception faite des valeurs rapportées par LANDA et al. [6] : ces dernières, nettement plus élevées, seraient toutefois attribuables à la méthode expérimentale [22], engendrant des conditions anoxiques, réductrices, qui provoqueraient la précipitation de formes réduites insolubles $\left(\mathrm{TcO}_{2}\right.$, sulfures, coprécipitation avec $\mathrm{FeS}$ ). 
Conformément à cette interprétation, les études de transfert de ${ }^{99} \mathrm{Tc}$ entre le sol et la plante ont montré que sa mobilité et sa bioassimilabilité sont gouvernées par le potentiel rédox du substrat [8 - 14].

En rizière irriguée par submersion, les conditions biochimicophysiques du sol sont modifiées par la présence d'une nappe d'eau entraînant, entr'autre, un retard des échanges gazeux : il s'ensuit, dès après la submersion, une forte diminution du potentiel d'oxydo-réduction qui atteint un minimum après quelques jours [17]; d'autre part, diverses études de radiopollution ont souligné la part prise par la base immergée des chaumes feuillés dans l'absorption des éléments minéraux $[2,10-12]$.

Dans ce contexte, l'étude de l'accumulation de ${ }^{99} \mathrm{~T} c$ par le riz en culture submergée trouve son réel intérêt. Des cinétiques d'absorption et de désorption racinaire en solution nutritive sous flux continu ont été réalisées concomitamment; les résultats acquis sont présentés ci-après et complètent les données obtenues en sol irrigué.

\section{MATÉRIEL ET MÉTHODES}

Le choix du matériel végétal s'est porté sur la variété de riz B 6044 caractérisée par un cycle court et un enracinement rapide et profond. Les graines sont mises à germer sur eau déminéralisée qui, avec le développement des plantules, est progressivement remplacée par une solution nutritive dilué dont la composition est la suivante (macroéléments en $\mu \mathrm{M}$ ) : $\mathrm{KH}_{2} \mathrm{PO}_{4} 32 ; \mathrm{KCl} 512 ; \mathrm{CaCl}_{2} .2 \mathrm{H}_{2} \mathrm{O} 2,5$; $\mathrm{MgSO}_{4} .7 \mathrm{H}_{2} \mathrm{O} 41$; EDTA Na-Fe 18; $\left(\mathrm{NH}_{4}\right)_{2} \mathrm{SO}_{4} 714$; et oligoéléments en $\mathrm{nM}: \mathrm{H}_{3} \mathrm{BO}_{3}$ 9,$88 ; \mathrm{MnCl}_{2} .4 \mathrm{H}_{2} \mathrm{O}$ 1,96; $\mathrm{ZnSO}_{4} .7 \mathrm{H}_{2} \mathrm{O} 1,93 ; \mathrm{CuSO}_{4} .5 \mathrm{H}_{2} \mathrm{O} 0,233,\left(\mathrm{NH}_{4}\right)_{6} \mathrm{Mo}_{7} \mathrm{O}_{24} .4 \mathrm{H}_{2} \mathrm{O}$ 0,$0275 ; \mathrm{pH} 5,8$.

\section{Etudes cinétiques}

A l'âge de $40 \mathrm{j}$, les plantes qui, depuis leur germination, ont été maintenues en lumière continue, sont transférées par groupe de cinq dans des colonnes de verre, d'une hauteur utile de $21 \mathrm{~cm}$ et d'un diamètre intérieur de $5,5 \mathrm{~cm}$, pourvues d'un siphon. Les racines sont soumises durant $72 \mathrm{~h}$ à un flux continu $\left(70 \mathrm{ml} . \mathrm{h}^{-1}\right) \mathrm{de}$ la solution nutritive décrite ci-dessus, contaminée par du $\mathrm{NH}_{4} \mathrm{TcO}_{4}$ aux concentra-

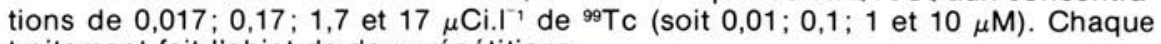
traitement fait l'objet de deux répétitions.

Après cette première phase d'absorption, les racines sont rincées à l'eau déminéralisée et les plantes replacées sur d'autres colonnes identiques à celles utilisées précédemment où elles sont soumises, 3 jours durant, à un flux continu de solution nutritive non contaminée à raison de $70 \mathrm{ml}^{-\mathrm{h}^{-1}}$.

Des quantités aliquotes de l'éluat sont prélevées à intervalles définis pour la détermination des teneurs en ${ }^{99} \mathrm{Tc}$ : les différences d'activités entre l'influx et l'efflux permettent le calcul des quantités de Tc absorbées ou désorbées. Durant les 144 heures de l'expérience, les plantes sont soumises à une lumière continue d'une intensité de $50 \mathrm{~W} \cdot \mathrm{m}^{-2}$.

\section{Etude du transfert sol-plante en culture irriguée}

35 jours après la germination, les plantules de riz sont repiquées deux par deux dans des colonnes cylindriques contenant $567 \mathrm{~g}$ de sol de Vercelli (Station expérimentale de riziculture de Vercelli, Italie), dont les principales caractéristiques sont reprises au tableau $\mathrm{I}$. 
TABLEAU I

Principales caractéristiques du sol de Vercelli

Texture (\% de la fraction minérale)

Argile $5,50 \%$

Limon .............. 22,40\%

Sable ..............69,10\%

Refus $(2 \mathrm{~mm}) \ldots \ldots \ldots .3,00 \%$
Matière organique .......... 2,32\%

Azote total ............... 1,49\%

$\mathrm{K}$ sous forme de $\mathrm{K}_{2} \mathrm{O} \ldots \ldots \ldots, 0,24 \%$

$P$ sous forme de $\mathrm{P}_{2} \mathrm{O}_{5} \ldots \ldots .1,68 \%$

$\mathrm{pH} \ldots \ldots \ldots \ldots \ldots \ldots \ldots, 5$

Les colonnes (h $29 \mathrm{~cm} \times \varnothing 5 \mathrm{~cm}$ ) sont recouvertes de papier aluminium et des granulés de PVC noir sont dispersés à la surface de l'eau d'irrigation, de manière à limiter la pénétration de la lumière et le développement d'algues. La contamination en Tc est réalisée par apport journalier, compensant en volume l'évapotranspiration, d'une solution nutritive diluée de composition décrite précédemment, additionnée de $\mathrm{NH}_{4} \mathrm{TcO}_{4}$ aux concentrations de 0,17 et $17 \mu \mathrm{Ci} . \mathrm{I}^{-1}$ de ${ }^{99} \mathrm{Tc}$, soit 0,1 et $10 \mu \mathrm{M}$. La figure 1 illustre l'apport cumulé de solution nutritive en fonction du temps pour chacun des traitements. Chaque traitement fait l'objet de deux répétitions.

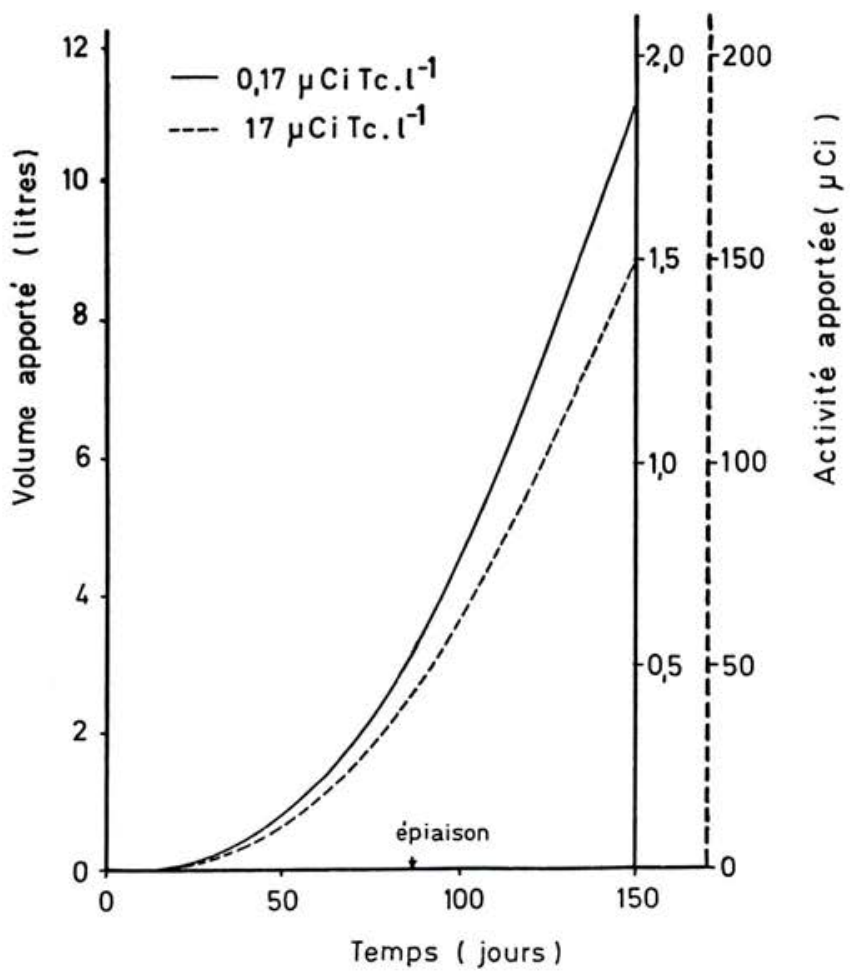

Fig. 1. - Apport cumulé en fonction du temps de solution nutritive contaminée en Tc (en volume et en activité) aux cultures de riz en sol irrigué par submersion pour les deux traitements envisagés. 
Depuis la germination jusqu'à la récolte, les plantes ont été soumises à une photopériode 12/12; l'intensité lumineuse étant de $50 \mathrm{~W} \cdot \mathrm{m}^{-2}$. A maturation $\left(150^{\circ}\right.$ jour), les plantes sont récoltées et séparées en racine, bases des chaumes, chaumes feuillés, panicules vides, graines stériles, balles et caryopses des graines fertiles. Des mesures de poids frais et de poids sec sont effectuées et des échantillons de chacune des fractions sont minéralisés en vue de la détermination de l'activité en ${ }_{99} \mathrm{TC}$ (digestion humide $\mathrm{HNO}_{3} 65 \%+\mathrm{H}_{2} \mathrm{O}_{2} 30 \%$ ).

\section{Détermination de l'activité en ${ }^{99} \mathrm{Tc}$}

Les prélèvements de solution nutritive $(5 \mathrm{ml})$ et les aliquotes $(5 \mathrm{ml})$ des minéralisats d'échantillons végétaux repris par $\mathrm{HNO}_{3} \quad 0,2 \mathrm{~N}$, sont additionnés à $5 \mathrm{ml}$ d'Instagel (Packard); l'activité en ${ }^{99} \mathrm{TC}$ est mesurée par scintillation liquide (Packard TRI-CARB 2450).

\section{Détermination des espaces libres apparents}

La quantité de Tc présente dans les espaces libres racinaires est déterminée à partir des courbes traduisant l'absorption et la désorption de cet élément en fonction du temps. En acceptant, a priori, que la concentration à l'intérieur de ces espaces soit égale à celle de la solution extérieure, on peut calculer leur volume. II est, cependant, à noter que cette hypothèse fait l'objet de sérieuses critiques qui sont à la base du concept d'espaces libres apparents [4].

\section{Analyse compartimentale}

Cette analyse repose sur la décomposition possible de la plante en divers compartiments disposés en série et/ou en parallèle, chacun étant caractérisé par une capacité apparente et une demi-résidence de l'élément étudié. Les résultats sont obtenus à partir des courbes retraçant en diagramme semi-logarithmique l'évolution décroissante de la teneur en ${ }^{99} \mathrm{TC}$ de la plante en fonction du temps.

\section{RÉSULTATS ET DISCUSSION}

\section{Cinétiques d'absorption et de désorption}

L'évolution de la teneur en ${ }^{99} \mathrm{~T} c$ des plantes de riz en fonction du temps et de la concentration en radionucléide est illustrée par la figure 2 . Les courbes présentent une première phase d'absorption rapide durant les quelques premières heures; ce phénomène peut être associé à un processus physique de diffusion et d'accumulation dans les espaces libres racinaires. Ce mécanisme cède progressivement le pas à une absorption plus lente se prolongeant de façon linéaire. Cette seconde phase trouve son interprétation dans la pénétration cellulaire, la translocation et l'accumulation du Tc dans la partie aérienne.

Le phénomène observé dans les limites des concentrations en Tc étudiées $\left(0,017\right.$ à $17 \mu \mathrm{Ci} . \mathrm{I}^{-1}$, soit 0,01 à $\left.10 \mu \mathrm{M}\right)$ peut être assimilé à une cinétique du type Michaelis-Menten dont les paramètres sont $\mathrm{Km}: 4,110^{-6} \mathrm{M}$ et $\mathrm{V} \max : 1,66 \mu \mathrm{M} \cdot \mathrm{g}^{-1}$ (M.S.R.). $\mathrm{h}^{-1 *}$.

Les courbes de désorption présentent les mêmes caractéristiques; la quantité de Tc désorbé durant la première phase $(\simeq 18 \mathrm{~h})$ ne représente que 4,3 à $4,6 \%$ du Tc absorbé durant une période de $72 \mathrm{~h}$, ce qui, en fonction des autres observations (tableau IV) indique que seul le Tc non fixé ou non métabolisé est désorbé (tableau II). La quantité totale de ${ }^{99} \mathrm{Tc}$ désorbé n'est guère plus élevée : elle varie de $7,23 \%$ $\left(0,017 \mu \mathrm{Ci} . \mathrm{I}^{-1}\right)$ à $11,57 \%\left(17 \mu \mathrm{Ci} . \mathrm{I}^{-1}\right)$.

$\overline{\text { M.S.R. }}=$ matière sèche racinaire. 

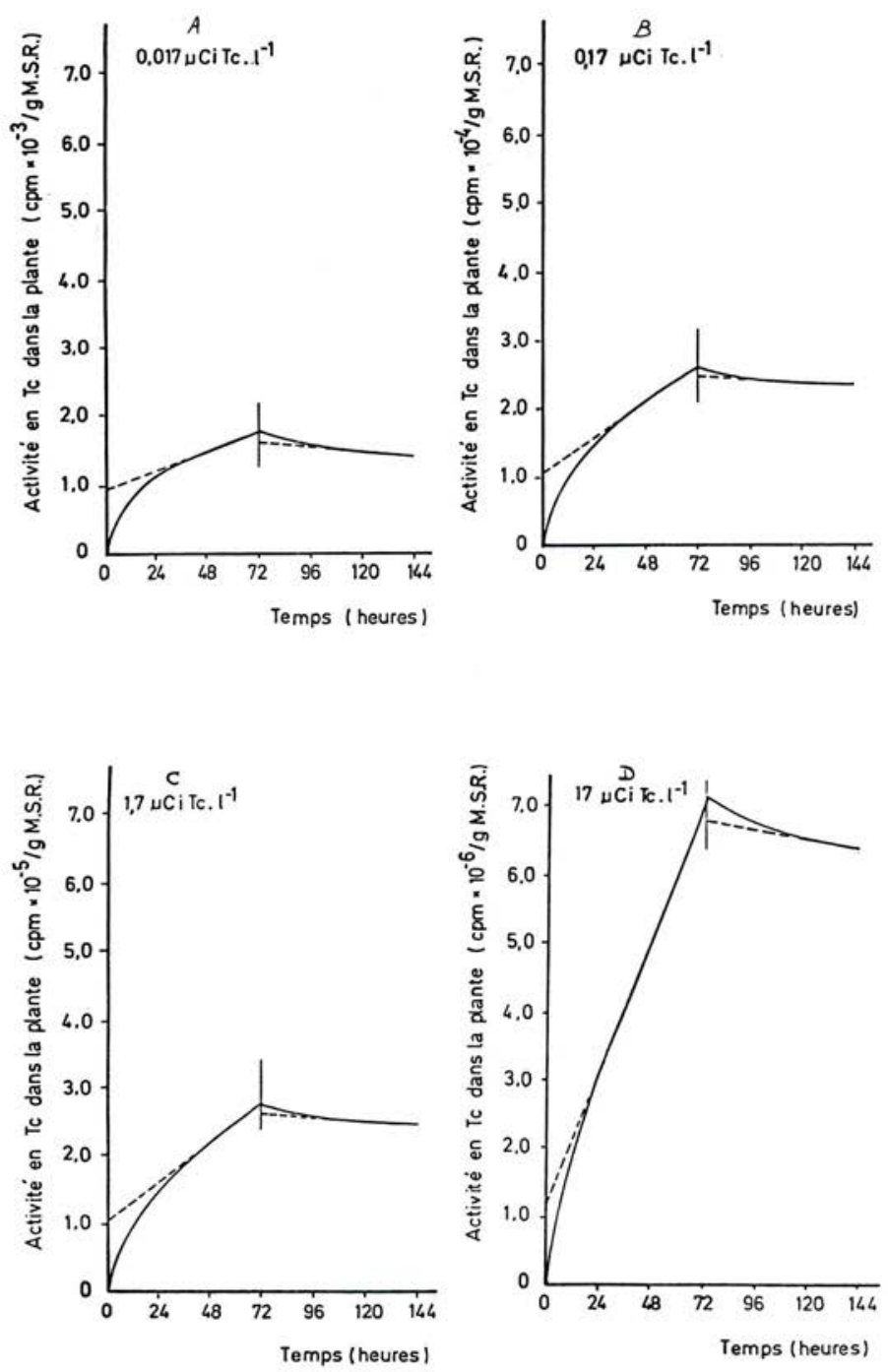

Fig. 2. - Absorption et désorption du Tc par le riz cultivé en flux continu de solution nutritive pour les quatre concentrations étudiées (A, B, C et D). 
TABLEAU ॥

Fraction (en $\%$ du contenu total de la plante après $72 \mathrm{~h}$ d'absorption) du technétium désorbé à la fin de la première phase et après $72 \mathrm{~h}$

\begin{tabular}{|l|c|c|c|c|}
\hline \multirow{2}{*}{ Phase } & \multicolumn{4}{|c|}{ Concentration initiale en Tc de la solution $\left(\mu \mathrm{Ci} . \mathrm{I}^{-1}\right)$} \\
\cline { 2 - 5 } & 0,017 & 0,17 & 1,7 & 17 \\
\hline $1^{\text {re }}$ Phase $(18 \mathrm{~h})$ & 4,61 & 4,55 & 4,31 & 4,26 \\
Désorption totale $(72 \mathrm{~h})$ & 7,23 & 7,81 & 8,51 & 11,57 \\
\hline
\end{tabular}

Le volume des espaces libres apparents racinaires a été calculé en absorption et en désorption; ce calcul repose sur l'assimilation de la phase passive de l'absorption à un phénomène de simple diffusion (tableau III). Dans chacun des cas, les volumes sont identiques quels que soient les traitements; cependant, on observe une différence marquée (facteur 10) entre les volumes calculés en absorption et en désorption.

\section{TABLEAU III}

Volumes des espaces libres racinaires apparents calculés pour les quatre concentrations en ${ }^{99} \mathrm{Tc}$ de la solution nutritive et en phases d'absorption et de désorption.

\begin{tabular}{|c|c|c|c|c|}
\hline \multirow{2}{*}{$\begin{array}{c}\text { Volumes des espaces libres } \\
\text { apparents }\left(\mathrm{ml}^{-1} \mathrm{~g}^{-1} \text { ) (MSR) }\right.\end{array}$} & \multicolumn{4}{|c|}{ Concentration initiale en Tc de la solution $\left(\mu \mathrm{Ci} . \mathrm{I}^{-1}\right)$} \\
\cline { 2 - 5 } & 0,017 & 0,17 & 1,7 & 17 \\
\hline en absorption & 29,2 & 29,0 & 30,7 & 28,2 \\
en désorption & 2,9 & 3,0 & 2,9 & 3,0 \\
\hline
\end{tabular}

Les mécanismes suivants peuvent être invoqués pour expliquer la différence observée :

- L'existence d'un espace libre de Donnan : l'équilibre de Donnan explique toutefois l'accumulation de cations, mais ne peut justifier celle des anions. Une modification de la forme chimique du Tc au niveau de la rhizosphère (réduction du $\mathrm{TCO}_{4}^{-}$) constitue un préalable nécessaire à tel comportement du Tc.

- L'immobilisation du pertechnétate dans les espaces libress par le truchement de la formation de composés se fixant dans ces espaces (réactions $\mathrm{TcO}_{4}{ }^{-}$composés soufrés du type cystéine).

- L'existence d'une certaine capacité d'échange anionique au niveau de l'apoplasme racinaire ce qui ne nécessiterait aucune transformation chimique préalable du $\mathrm{TcO}_{4}{ }^{-}$. PETTERSON [16] et PERSSON [15] ont démontré l'existence dans les racines de sites positifs responsables de liaison labiles avec les sulfates; la localisation la plus probable de ces sites d'échange serait la surface externe du plasmalemme.

L'analyse compartimentale (figure $3: 1,7 \mu \mathrm{Ci} . \mathrm{I}^{-1}$ de ${ }^{99} \mathrm{TC}$ ) révèle l'existence de deux compartiments dont les paramètres sont repris au tableau IV pour les différentes concentrations étudiées. La capacité apparente du premier compartiment 
exprimée en $\mathrm{nM}$ est, si l'on tient compte des concentrations en ${ }^{99} \mathrm{~T} c$ des solutions, identique pour les quatre concentrations étudiées. Cette première observation souligne la crédibilité des résultats présentés. De plus, la comparaison de ces données aux valeurs traduisant le volume des espaces libres calculés en désorption (tableau III) indique, comme l'on pouvait s'y attendre, que ce premier compartiment correspond aux espaces ouverts à toute diffusion libre des ions.

Le deuxième compartiment qui contient plus de $95 \%$ du Tc prélevé par la plante, se caractérise également par une demi-résidence nettement supérieure à celle du premier compartiment. Ce dernier résultat implique une décontamination potentielle très faible des plantes de riz suite à une pollution accidentelle des rizières.

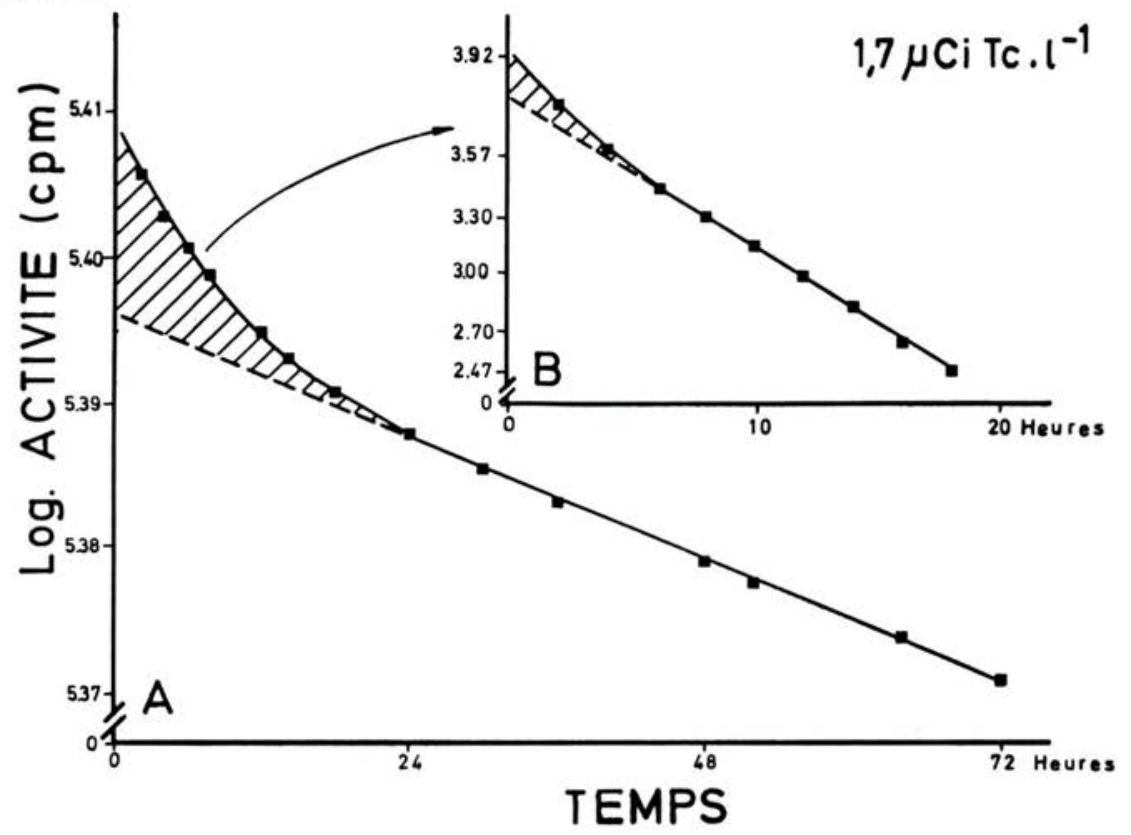

Fig. 3. - Analyse compartimentale de la distribution du Tc chez le riz (traitement 1,7 $\mu \mathrm{Ci} . \mathrm{I}^{\prime}$ ).

On remarque, par ailleurs, que la capacité du second compartiment, correspondant à la fraction du technétium fixé de façon plus ou moins irréversible dans les racines ou ayant été absorbé par la plante, est semblable pour les trois concentrations les plus faibles, compte tenu de la teneur en technétium dans la solution contaminée; les facteurs de transfert pour ces trois traitements sont donc identiques ou décroissent légèrement avec l'augmentation du niveau de contamination [18].

A la concentration la plus élevée $\left(17 \mu \mathrm{Ci} . \mathrm{I}^{-1}\right)$, la capacité du second compartiment et le facteur de transfert augmentent d'un facteur 2 , résultante probable d'un phénomène de toxicité. Cette observation, qui se rapporte à des conditions d'aquiculture, ne se vérifie toutefois pas en présence d'une phase solide [8] comme le montrent également les résultats de l'essai en sol décrit ci-après. 
TABLEAU IV

Analyse compartimentale : valeurs des paramètres caractéristiques des différents compartiments pour les différentes concentrations en ${ }^{99} \mathrm{Tc}$ - capacité apparente (C) exprimée en $\mathrm{nM}$ et demi-résidence (T 1/2) exprimée en heure.

\begin{tabular}{|c|c|c|c|c|c|c|c|c|}
\hline & \multicolumn{8}{|c|}{ Concentration en ${ }^{99} \mathrm{Tc}\left(\mu \mathrm{Ci} . \mathrm{I}^{-1}\right)$ pour l'absorption } \\
\hline & \multicolumn{2}{|c|}{0,017} & \multicolumn{2}{|c|}{0,17} & \multicolumn{2}{|c|}{1,7} & \multicolumn{2}{|c|}{17} \\
\hline & C & T $1 / 2$ & C & T $1 / 2$ & C & T $1 / 2$ & C & $\mathrm{T} 1 / 2$ \\
\hline $1^{\text {er }}$ comp. & 0,0341 & 3,84 & 0,338 & 3,62 & 3,04 & 4,25 & 25,06 & 4,41 \\
\hline $2^{\mathrm{e}} \mathrm{comp}$. & 0,778 & 1198 & 7,63 & 1193 & 72,4 & 925 & 1973 & 557 \\
\hline
\end{tabular}

\section{Etude du transfert du technétium en sol irrigué par submersion}

Les plantes irriguées par submersion à l'aide de la solution la plus concentrée en ${ }^{99} \mathrm{Tc}\left(17 \mu \mathrm{Ci} . \mathrm{I}^{-1}\right)$ ont manifesté certains symptômes de toxicité (tableau V) : les plantes sont de plus petite taille, le matériel végétatif aérien plus hydraté (retard de maturation?) et les rendements en graines fortement réduits. Ces résultats s'accompagnent d'une distribution relative différente du technétium (tableau VI) entre les divers organes de la plante.

Les données présentées indiquent que, pour les deux niveaux de Tc envisagés, plus de $90 \%$ du Tc absorbé par la plante se localise dans la partie aérienne végétative. Ce résultat confirme les résultats d'autres études menées en nos laboratoires et à l'extérieur [9-18]; $1 \%$ seulement du Tc absorbé se localise dans les caryopses (partie comestible).

\section{TABLEAU V}

Poids sec et teneur en eau des différents organes en fonction de la concentration en ${ }^{99} \mathrm{Tc}$ dans l'eau d'irrigation.

PS $=$ poids sec, $\% \mathrm{H}_{2} \mathrm{O}=$ teneur en eau.

\begin{tabular}{|c|c|c|c|c|c|c|}
\hline \multirow{3}{*}{ Organe } & \multicolumn{6}{|c|}{ Concentration en Tc $\left(\mu \mathrm{Ci} . I^{\top}\right)$} \\
\hline & \multicolumn{2}{|c|}{0,00} & \multicolumn{2}{|c|}{0,17} & \multicolumn{2}{|c|}{17,00} \\
\hline & PS & $\% \mathrm{H}_{2} \mathrm{O}$ & PS & $\% \mathrm{H}_{2} \mathrm{O}$ & PS & $\% \mathrm{H}_{2} \mathrm{O}$ \\
\hline Racines & 5,7 & 66,5 & 4,9 & 66,4 & 4,8 & 66,5 \\
\hline Parties aériennes végétatives & 11,5 & 68,1 & 10,2 & 70,6 & 7,2 & 77,2 \\
\hline Graines fertiles & 14,0 & 15,3 & 12,0 & 10,3 & 3,1 & 17,8 \\
\hline Graines stériles & 1,7 & 28,2 & 1,7 & 33,7 & 0,2 & 40,0 \\
\hline Plante entière & 32,9 & 54,3 & 28,8 & 55,9 & 15,1 & 69,5 \\
\hline
\end{tabular}




\section{TABLEAU VI}

Distribution relative (en \%) du technétium dans les différents organes de la plante.

\begin{tabular}{|c|c|c|}
\hline \multirow{2}{*}{ Organe } & \multicolumn{2}{|c|}{$\begin{array}{c}\text { Concentration en Tc dans l'eau } \\
\text { d'irrigation }\left(\mu \mathrm{Ci} . I^{-1}\right)\end{array}$} \\
\hline & 0,17 & 17,00 \\
\hline Racines & $\begin{array}{r}1,2 \\
05\end{array}$ & $\begin{array}{r}3,3 \\
035\end{array}$ \\
\hline Bases des chaumes & 59.6 & 55.6 \\
\hline Chaumes feuillés & 33,8 & 37,0 \\
\hline Panicules vides & 1,7 & 0,8 \\
\hline Graines fertiles & 3,4 & 3,2 \\
\hline Balles & 2,6 & 2,1 \\
\hline Caryopses & 0,9 & 1,0 \\
\hline Graines stériles & 0,3 & 0,0 \\
\hline
\end{tabular}

Les facteurs de transfert entre la solution d'irrigation et les plantes de riz sont donnés au tableau VII. Ces facteurs, bien que variant en fonction de la teneur en ${ }^{99} \mathrm{Tc}$ de la solution sont extrêmement élevés et dépassent de loin ceux obtenus pour la majorité des autres radioisotopes étudiés [17]. Pour la plus faible concentration $\left(0,17 \mu \mathrm{Ci} . \mathrm{I}^{-1}\right)$ les valeurs atteignent respectivement $\simeq 1000$ pour les chaumes feuillés et $\simeq 50$ pour les graines fertiles. Les chiffres très élevés obtenus pour la base des chaumes laissent supposer une absorption très forte à ce niveau suivie d'une translocation importante vers les feuilles et, dans une moindre mesure, vers les organes génératifs. Les mêmes observations peuvent être faites pour la concentration la plus élevée.

TABLEAU VII

Facteur de transfert eau d'irrigation/plante (estimé par rapport au poids sec).

\begin{tabular}{|c|c|c|}
\hline \multirow{2}{*}{ Organe } & \multicolumn{2}{|c|}{$\begin{array}{l}\text { Concentration en Tc de la solution } \\
\left(\mu \mathrm{Ci} . I^{-1}\right)\end{array}$} \\
\hline & 0,17 & 17,00 \\
\hline Racines & 45 & 18 \\
\hline Parties aériennes végétatives & 1656 & 342 \\
\hline Base des chaumes & 2819 & 556 \\
\hline Chaumes feuillés & 1054 & 239 \\
\hline Panicules vides & 411 & 43 \\
\hline Graines fertiles & 51 & 27 \\
\hline Graines stériles & 33 & 3 \\
\hline Plante entière & 619 & 173 \\
\hline
\end{tabular}




\section{CONCLUSION}

Des résultats présentés ci-avant, il ressort que le problème de contamination de cultures irriguées par submersion qui, a priori, (réduction du $\mathrm{TcO}_{4}{ }^{-}$en sol inondé) aurait dû se poser d'une manière moins critique, conserve toute son importance du fait de l'absorption directe par la base des chaumes.

Par ailleurs, l'accumulation importante observée dans les parties aériennes, graines et chaumes, pose divers problèmes quant à leur utilisation tant pour l'alimentation humaine qu'au niveau des différentes pratiques agronomiques (incinération des chaumes sur le champs, utilisation des pailles en alimentation du bétail ou comme litière avec perspective de recyclage par épandage du fumier).

\section{BIBLIOGRAPHIE}

[1] BITTEL R. Le technétium et l'environnement. Radioprotection, 1980, 15, 141-146.

[2] BOURDEAU Ph., CAVALLORO R., MYTTENAERE C. et VERFAILliE G. Movement of fallout radionuclides in ecosystems of the Po valley, Italy. Health Phys., 1965, 11, $1429-1444$.

[3] CATALDO D.A., WILDUNG R.E. et GARLAND T.R. Technetium accumulation, fate and behaviour in plants. In : Proceedings of the symposium on environmental chemistry and cycling processes, Augusta, April 27-30, 1976. CONF-760429, 1978, p. 28.

[4] HELLER R. Les mécanismes de l'absorption. In : Biologie végétale, Vol. II Nutrition et métabolisme. Paris : Masson, 1969, 339-357.

[5] JOHNSTON H.M. et GILLHAM R.W. Technetium. In : A review of selected radionuclide distribution coefficients of geologic materials. Atomic Energy of Canada Limited, Research Compagny, TR-90, 1980, 51-55.

[6] LANDA E.R., HART-THORVIG L. et GAST R.G. Effect of selective dissolution, electrolytes, aeration and sterilization on technetium 99 sorption by soils. J. Environ. Qual., 1977, 6, 181-187.

[7] MENZEL R.G. Soil-plant relationships of radioactive elements. Health Phys., $1965,11,1325-1332$.

[8] MOUSNY J.M. et MYTTENAERE C. Absorption of technetium by plants in relation to soil type, contamination level and time. Plant and Soil, 1981, 61, 403-412.

[9] MOUSNY J.M., ROUCOUX P. et MYTTENAERE C. Absorption and translocation of technetium in pea plant. Env. Exp. Bot., 1979, 19, 263-268.

[10] MYTTENAERE C. Absorption of radiocaesium by flooded rice : relative importance of roots and shoot bases in the transfer of radioactivity. Plant and Soil, 1972, 36, 215-218.

[11] MYTTENAERE $\mathrm{C}$. et BOURDEAU Ph. Transfert des radionucléides en rizières irriguées. Giorn. Fis. San. Radioprot., 1968, 12, 179-181.

[12] MYTTENAERE C., BOURDEAU Ph. et MASSET M. Relative importance of soil and water in the indirect contamination of flooded rice with radiocaesium. Health Phys., 1969, 16, 701-707.

[13] MYTTENAERE C., DAOUST C. et ROUCOUX P. Leaching of technetium from foliage by simulated rain. Env. Exp. Bot., 1980, 20, 415-419.

[14] PAQUETTE J., REID J.A.K. et ROSINGER E.L.J. Review of technetium behaviour in relation to nuclear waste disposal. Atomic Energy of Canada Limited, Research Company, TR-25, 1980, 1-25. 
[15] PERSSON L. Labile-bound sulfate in wheat-roots : localization, nature and possible connection to the active absorption mechanism. Physiol. Plant., 1969, 22, 959-976.

[16] PETTERSON S. Active and passive components of sulfate uptake in sunflower plants. Physiol. Plant., 1966, 19, 459-492.

[17] PONNAMPER $U$ MA F.N. The chemistry of submerged soils in relation to the growth and yield of rice. Unpublished Ph. D. Thesis, Cornell University, Ithaca, N.-Y., 1955, 208 p., In : PONNAMPERUMA F.N. Dynamic aspects of flooded soils and the nutrition of the rice plant, proceedings Symposium, International Rice Research Institute. The mineral nutrition of the rice plant, Los Banos, Laguma (Philippines), Feb. 1964. Baltimore: J. Hopkins press, 1965, 295-328.

[18] ROUCOUX P. Etude du comportement du Technétium chez le soja en milieu contaminé ou non en cadmium. Thèse de doctorat, Faculté des Sciences, Lab. Physiol. Vég., U.C.L., Louvain-la-Neuve, 1980, 127 p.

[19] SAAS A., DENARDI J.L., COLLE E. et QUINAULT J.M. Cycle du molybdène et du technétium dans l'environnement. Evolution physicochimique et mobilité dans les sols et les végétaux. In : $2^{\text {e }}$ Symposium International de radioécologie, Cadarache, 19-22 juin 1979, 443-489.

[20] TILL J.E., HOFFMAN F.O. et DUNNIG D.E.Jr. A new look at ${ }^{99} \mathrm{TC}$ releases to the atmosphere. Health Phys., 1979, 36, 21-30.

[21] WILDUNG R.E., GARLAND T.R. et CATALDO D.A. Accumulation of technetium by plants. Health Phys., 1977, 32, 314-317.

[22] WILDUNG R.E., McFADDEN K.M. et GARLAND T.R. Technetium sources and behaviour in the environment. J. Environ. Qual., 1979, 8, 156-161.

[23] WILDUNG R.E., ROUTSON R.C., SERNE R.J. et GARLAND T.R. Pertechnetate, iodide and methyl iodide retention by surface soils. USERDA Report BNWL-1950, Part 2, 1974, 37-41. 